hep-th/0408154

BROWN-HET-1422

\title{
Perturbations in a Holographic Universe and in Other Stiff Fluid Cosmologies
}

\author{
T. J. Battefeld 1)* and D. A. Easson 2) $\dagger$ \\ 1) Physics Department, Brown University, Providence, RI 02912, USA. \\ 2) Department of Physics, Syracuse University, Syracuse, NY 13244-1130, USA.
}

(Dated: November 23, 2018)

\begin{abstract}
We examine the generation and evolution of perturbations in a universe dominated by a fluid with stiff equation of state $p=\rho$. The recently proposed Holographic Universe is an example of such a model. We compute the spectrum of scalar and tensor perturbations, without relying on a microphysical description of the $p=\rho$ fluid. The spectrum is scale invariant deep inside the Hubble horizon. In contrast, infrared perturbations that enter the Hubble horizon during the stiff fluid dominated (holographic) phase yield oscillatory and logarithmic terms in the power spectrum. We show that vector perturbations grow during the stiff fluid dominated epoch and may result in a turbulent and anisotropic Universe at the end of the holographic phase. Therefore, the required period of inflation following the holographic phase cannot be much shorter than that required in standard inflationary models.
\end{abstract}

PACS numbers: 98.80.Cq.

\section{INTRODUCTION}

Over the last few years many attempts have been made to merge string theory with cosmology. These models incorporate certain aspects of string theory like extra dimensions, branes, T-duality etc., and often drastically change our picture of the early Universe. Some popular examples are the KKLMMT model [1], the Cyclic/Ekpyrotic model 22, 3], Brane Gas Cosmology [4, [5, 6], Randall-Sundrum phenomenology [7, 8] and the Pre-Big-Bang scenario [9, 10] [54]. Ultimately, these models must make contact with experimental observation; one of the most demanding being the observation of a nearly scale invariant spectrum of density fluctuations [19]. So far, these models rely on inflation (in some form or another) in order to generate such a spectrum.

Recently, a radical new model based on the holographic principle of 't Hooft and Susskind 20, 21] was proposed by Banks and Fischler (BF) 22, 23, 24, 25, 26]. In this model the initial state of the Universe is described by a stiff fluid (with equation of state $p=\rho$ ) that saturates the holographic covariant entropy bound. The model conforms with standard cosmology (obeys the Einstein equations with Friedmann-Robertson-Walker metric) with this unusual form of matter. While we focus on the Holographic Universe model, a number of authors have considered cosmological aspects of such a stiff fluid in the literature and our conclusions generalize to those models as well [27]-37], 55]. The microphysical interpretation of this fluid is a dense gas of black holes [23] which is argued by BF to emerge naturally from a quantumgravity regime.

It is claimed that, during the holographic phase, a scale invariant spectrum of perturbations is generated inside

\footnotetext{
*battefeld@physics.brown.edu

†easson@physics.syr.edu
}

the Hubble horizon. Thereafter, it is inflated to superHubble scales during a short burst of inflation [23]. A careful calculation of the spectrum and an implementation of the inflationary phase needs to be performed [56].

In this article, we address the first of these issues and compute the spectrum of scalar and tensor perturbations inside the Hubble horizon. The spectrum originates from sub-Hubble ultraviolet (UV) perturbations, and infrared (IR) perturbations that crossed the Hubble radius during the holographic phase. We find that UV perturbations do indeed yield a scale invariant spectrum; however, IR perturbations have oscillatory and logarithmic corrections to scale invariance.

Vector perturbations (VP) are found to grow during the holographic phase. We argue that the Universe will become turbulent and anisotropic if the $p=\rho$ fluid dominates for a long enough time. We speculate that it might be this feature that puts an end to the holographic phase, since the Universe is no longer describable by an effective $p=\rho$ fluid.

Our main conclusion is that due to the imprints of IR perturbations, a rather long phase of inflation is required to make the holographic model consistent with observations. Although the holographic cosmology proposal does not eliminate the need for a period of cosmological inflation, there is hope that it will help resolve the initial singularity [26, 39].

\section{BACKGROUND}

We work with mostly negative signature $(+,-,-,-)$, scale factor $a(\eta)$, conformal time $\eta$ and we assume a flat, Friedmann-Roberston-Walker Universe, so that

$$
d s^{2}=a^{2} d \eta^{2}-a^{2} \delta_{i j} d x^{i} d x^{j} .
$$

The matter content is modelled by an ideal fluid

$$
T_{\beta}^{\alpha}=(\rho+p) u^{\alpha} u_{\beta}-p \delta_{\beta}^{\alpha},
$$


where $p$ is the fluid's pressure, $\rho$ is its energy density, and $u^{\alpha}$ is its four velocity satisfying $u^{\alpha} u_{\alpha}=1$ (given in a comoving frame by $\left.\left(u_{\alpha}\right)=(a, 0,0,0)\right)$. The unperturbed Einstein equations read

$$
G_{\beta}^{\alpha}=\frac{1}{k_{4}^{2}} T_{\beta}^{\alpha},
$$

where $k_{4}^{-2}=8 \pi G$, so that

$$
\begin{aligned}
2 \frac{a^{\prime \prime}}{a}-\left(\frac{a^{\prime}}{a}\right)^{2} & =-\frac{a^{2} p}{k_{4}^{2}}, \\
3\left(\frac{a^{\prime}}{a}\right)^{2} & =\frac{a^{2} \rho}{k_{4}^{2}},
\end{aligned}
$$

yielding the well known relation $\rho \sim a^{-3(1+w)}$ for the equation of state $p=w \rho$. In the above and throughout, prime denotes differentiation with respect to conformal time $\eta$.

The Holographic Universe model [22, 23, 24, 25, 26] is characterized by an equation of state parameter $w=1$ so that

$$
\begin{aligned}
& a(\eta)=\sqrt{\eta}, \\
& \rho(\eta)=\rho_{0} \eta^{-3} .
\end{aligned}
$$

The microphysical interpretation of such a fluid is involved, but is not required in the following analysis [57].

\section{SCALAR PERTURBATIONS}

\section{A. Metric}

In the longitudinal gauge the most general perturbed metric is given by

$$
d s^{2}=a^{2}\left[(1+2 \Phi) d \eta^{2}-(1-2 \Psi) \delta_{i j} d x^{i} d x^{j}\right] .
$$

Note that the two Bardeen potentials agree with the gauge invariant scalar metric perturbations - there is no residual gauge freedom. Since we do not consider anisotropic stress, the off-diagonal Einstein Equations yield

$$
\Phi=\Psi
$$

so that only one scalar metric degree of freedom remains.

\section{B. Matter and Metric}

Since we do not want to rely on a microphysical realization of the holographic matter, it is not straightforward to identify the gauge invariant, scalar degree of freedom, once matter is added. Fortunately, this difficult enterprise has already been carried out in [40], yielding the single gauge invariant degree of freedom

$$
v=\frac{1}{\sqrt{6} l}\left(\phi_{v}-2 z \Psi\right),
$$

where $l=\sqrt{8 \pi G / 3}, \phi_{v}$ is the velocity potential for the perturbation of the four velocity $\delta u_{i}$, that is

$$
\phi_{v, i}=-\frac{2 \sqrt{\beta} a^{2}}{c_{s}} \delta u_{i}
$$

and $z$ is given by

$$
\begin{aligned}
z & =\frac{a \sqrt{\beta}}{\mathcal{H} c_{s}}, \\
c_{s} & =\frac{p^{\prime}}{\rho^{\prime}}, \\
\beta & =\mathcal{H}^{2}-\mathcal{H}^{\prime},
\end{aligned}
$$

where $\mathcal{H}=a^{\prime} / a$. Thus with $p=\rho$ and our background solution (6) we have

$$
\begin{aligned}
\beta & =\frac{3}{4 \eta^{2}}, \\
c_{s} & =1 \\
z & =\sqrt{3 \eta} .
\end{aligned}
$$

The advantage of $v$ lies in the fact that its action takes the simple form of a scalar field with time dependent mass

$$
S_{v}=\frac{1}{2} \int d^{4} x\left(v^{\prime 2}-\delta^{i j} v_{, i} v_{, j}+\frac{z^{\prime \prime}}{z} v^{2}\right),
$$

and thus $v$ can be quantized in a straightforward way [40].

\section{SCALAR PERTURBATIONS IN THE $p=\rho$ ERA}

Ultimately, we are interested in the power spectrum

$$
\left|\delta_{\Phi}(\eta, k)\right|=\left(\frac{3 l^{2}}{8 \pi^{2}}\right)^{1 / 2} \frac{\sqrt{\beta} z}{a c_{s}} \frac{1}{\sqrt{k}}\left|\left(\frac{v_{k}(\eta)}{z}\right)^{\prime}\right|,
$$

where the mode functions $v_{k}(\eta)$ satisfy the equation of motion derived from (18) (in momentum space)

$$
v_{k}^{\prime \prime}+\left(c_{s}^{2} k^{2}-\frac{z^{\prime \prime}}{z}\right) v_{k}=0 .
$$

For our specific background the general solution is

$$
v_{k}(\eta)=A_{k} \sqrt{\eta} J_{0}(\eta k)+B_{k} \sqrt{\eta} Y_{0}(\eta k)
$$

where $A_{k}$ and $B_{k}$ are determined by initial conditions and $J_{n}$ and $Y_{n}$ are Bessel functions. We assume the fluctuations are seeded by quantum vacuum fluctuations and adopt the following general initial conditions that define the vacuum at $\eta=\eta_{i}$

$$
\begin{aligned}
& v_{k}\left(\eta_{i}\right)=k^{-1 / 2} M\left(k \eta_{i}\right), \\
& v_{k}^{\prime}\left(\eta_{i}\right)=k^{1 / 2} i N\left(k \eta_{i}\right),
\end{aligned}
$$


where $N$ and $M$ obey $N M^{\star}+N^{\star} M=2$ and the conditions

$$
\begin{aligned}
\left|M\left(k \eta_{i}\right)\right| & \rightarrow 1, \\
\left|N\left(k \eta_{i}\right)\right| & \rightarrow 1,
\end{aligned}
$$

for $k \eta_{i} \gg 1$. Note that the short wavelength behavior is generic for most choices of the vacuum.

\section{A. Short Wavelength}

For the short wavelength part of the spectrum, that is $k \eta \gg 1$, the Bessel functions can be expanded

$$
\begin{aligned}
J_{0}(k \eta) & \simeq \sqrt{\frac{2}{\pi k \eta}} \cos (k \eta-\pi / 4)+\mathcal{O}\left(\frac{1}{k \eta}\right), \\
Y_{0}(k \eta) & \simeq \sqrt{\frac{2}{\pi k \eta}} \sin (k \eta-\pi / 4)+\mathcal{O}\left(\frac{1}{k \eta}\right) .
\end{aligned}
$$

Using (21) and the initial conditions (22)-(23) the mode functions are given by

$$
v_{k}(\eta)=-\frac{i N}{\sqrt{k}} \cos \left(k\left(\eta-\eta_{i}\right)\right)-\frac{M}{\sqrt{k}} \sin \left(k\left(\eta-\eta_{i}\right)\right) .
$$

With this, the background solution (6) and (16)-(17) the spectrum (19) is

$$
\begin{aligned}
\left|\delta_{\Phi}(\eta, k)\right| & =\sqrt{\frac{9}{32}} \frac{l}{\pi} \times \\
& \mid \frac{1}{\eta^{3 / 2}}\left(M \cos \left(k\left(\eta-\eta_{i}\right)\right)-i N \sin \left(k\left(\eta-\eta_{i}\right)\right)\right) \\
& +\frac{1}{6 k \eta^{5 / 2}}\left(i N \cos \left(k\left(\eta-\eta_{i}\right)\right)+M \sin \left(k\left(\eta-\eta_{i}\right)\right)\right) \mid .
\end{aligned}
$$

If we focus on the dominant solution $\sim \eta^{-3 / 2}$, we see that the spectrum has a scale invariant shape, since the initial conditions (24) and (25) give

$$
\left|\delta_{\Phi}(\eta, k)\right|=\sqrt{\frac{9}{32}} \frac{l}{\pi \eta^{3 / 2}} .
$$

Note that this is essentially the spectrum that is generated during inflation inside the Hubble horizon 40].

So far, we have only considered the short wavelength fluctuations. However, in the stiff fluid dominated phase,
IR fluctuations are entering the Hubble horizon. If a subsequent inflationary phase lasts for only a few e-foldings, these primordial IR perturbations can leave an imprint on the CMBR.

\section{B. Long wavelength}

To discuss the long wavelength perturbations we need the general solution (21). To compute the coefficients $A_{k}$ and $B_{k}$ we must choose quantum initial conditions. However, this task is not as straightforward as in the short wavelength case, since there are ambiguities associated to the choice of vacuum 41]. Therefore, we will consider two general choices:

1. The same initial conditions as for the short wavelength perturbations (22) and (23)

$$
\begin{aligned}
& v_{k}\left(\eta_{i}\right)=k^{-1 / 2}, \\
& v_{k}^{\prime}\left(\eta_{i}\right)=i k^{1 / 2},
\end{aligned}
$$

this is a sensible choice (e.g. for non-exotic matter with $p \leq \rho / 3$, since the oscillator (20) becomes tachyonic in that case; see [4] for more details).

2. Realizing that $-z^{\prime \prime} / z=1 / 4 \eta^{2}>0$, we can straightforwardly quantize the oscillator [40], yielding

$$
\begin{aligned}
& v_{k}\left(\eta_{i}\right)=E^{-1 / 2}=\left(2 \eta_{i}\right)^{1 / 2}, \\
& v_{k}^{\prime}\left(\eta_{i}\right)=i E^{1 / 2}=i\left(2 \eta_{i}\right)^{-1 / 2},
\end{aligned}
$$

where we took the "energy" as $E^{2}=-z^{\prime \prime} / z$ in the limit $k \eta_{i} \ll 1$, with $z=\sqrt{3 \eta}$.

Computing $A_{k}$ and $B_{k}$ is then straightforward. Now one can compute an exact analytic expression for the spectrum of pertrubations (19) - note that there is no matching condition required, as we know the exact solution to (20). However, since the exact expression is quite cumbersome, we shall also provide an approximate solution valid for $k \eta_{i} \ll 1$ and $k \eta \gg 1$; this approximation is good for the spectrum of perturbations (19) inside the Hubble radius originating from IR perturbations that crossed the horizon during the holographic $(p=\rho)$ phase. With $\tau \equiv k \eta$ and $\tau_{i} \equiv k \eta_{i}$ we find 


$$
\begin{aligned}
\left|\delta_{\Phi}^{(1)}(\eta, k)\right|= & \frac{3 \sqrt{2}}{16} \frac{l}{\pi} \frac{k^{3 / 2}}{\tau_{i}^{3 / 2} \tau}\left|J_{1}\left(\tau_{i}\right) Y_{0}\left(\tau_{i}\right)-Y_{1}\left(\tau_{i}\right) J_{0}\left(\tau_{i}\right)\right|^{-1}\left[\left[J_{1}(\tau)\right]^{2}\left[Y_{0}\left(\tau_{i}\right)\right]^{2}+\left[Y_{1}(\tau)\right]^{2}\left[J_{0}\left(\tau_{i}\right)\right]^{2}\right. \\
& +4 \tau_{i}\left(\left[J_{1}(\tau)\right]^{2} Y_{0}\left(\tau_{i}\right) Y_{1}\left(\tau_{i}\right)+J_{1}(\tau) Y_{1}(\tau)\left[J_{0}\left(\tau_{i}\right) Y_{1}\left(\tau_{i}\right)+J_{1}\left(\tau_{i}\right) Y_{0}\left(\tau_{i}\right)\right]+\left[Y_{1}(\tau)\right]^{2} J_{0}\left(\tau_{i}\right) J_{1}\left(\tau_{i}\right)\right) \\
& \left.+4 \tau_{i}^{2}\left(\left(J_{1}(\tau) Y_{0}\left(\tau_{i}\right)-Y_{1}(\tau) J_{0}\left(\tau_{i}\right)\right)^{2}+\left(J_{1}(\tau) Y_{1}\left(\tau_{i}\right)-Y_{1}(\tau) J_{1}\left(\tau_{i}\right)\right)^{2}\right)\right]^{1 / 2} \\
\simeq & \frac{3}{16} \frac{l}{\pi^{3 / 2}} \frac{1}{\eta^{3 / 2}} \frac{1}{\sqrt{k \eta_{i}}}\left|2 \cos \left(k \eta+\frac{\pi}{4}\right)\left[\gamma+\ln \left(\frac{k \eta_{i}}{2}\right)+2\right]-\pi \sin \left(k \eta+\frac{\pi}{4}\right)\right|, \\
\left|\delta_{\Phi}^{(2)}(\eta, k)\right|= & \frac{3 \sqrt{2}}{8} \frac{l}{\pi} \frac{k^{3 / 2}}{\tau_{i} \tau}\left|J_{1}\left(\tau_{i}\right) Y_{0}\left(\tau_{i}\right)-Y_{1}\left(\tau_{i}\right) J_{0}\left(\tau_{i}\right)\right|^{-1}\left[\left[J_{1}(\tau)\right]^{2}\left[Y_{0}\left(\tau_{i}\right)\right]^{2}+\left[Y_{1}(\tau)\right]^{2}\left[J_{0}\left(\tau_{i}\right)\right]^{2}\right. \\
& -2 \tau_{i}\left(\left[J_{1}(\tau)\right]^{2} Y_{0}\left(\tau_{i}\right) Y_{1}\left(\tau_{i}\right)-J_{1}(\tau) Y_{1}(\tau)\left[J_{0}\left(\tau_{i}\right) Y_{1}\left(\tau_{i}\right)+J_{1}\left(\tau_{i}\right) Y_{0}\left(\tau_{i}\right)\right]+\left[Y_{1}(\tau)\right]^{2} J_{0}\left(\tau_{i}\right) J_{1}\left(\tau_{i}\right)\right) \\
& \left.+2 \tau_{i}^{2}\left(J_{1}(\tau) Y_{1}\left(\tau_{i}\right)-Y_{1}(\tau) J_{1}\left(\tau_{i}\right)\right)^{2}\right]^{1 / 2} \\
\simeq & \frac{3}{16} \frac{l}{\pi^{3 / 2}} \frac{1}{\eta^{3 / 2}} \sqrt{2}\left[\left(2 \cos \left(k \eta+\frac{\pi}{4}\right)\left[\gamma+\ln \left(\frac{k \eta_{i}}{2}\right)+2\right]-\pi \sin \left(k \eta+\frac{\pi}{4}\right)\right)^{2}\right. \\
& \left.+\left(2 \cos \left(k \eta+\frac{\pi}{4}\right)\left[\gamma+\ln \left(\frac{k \eta_{i}}{2}\right)\right]-\pi \sin \left(k \eta+\frac{\pi}{4}\right)\right)^{2}\right]^{1 / 2},
\end{aligned}
$$

where the superscript ${ }^{(1,2)}$ denotes the choice of the initial conditions and $\gamma$ is Euler's constant. Clearly, the specturm is not scale invariant.

\section{Discussion}

Comparing (36) and (38) with (30) we see that IR perturbations do not yield a scale invariant spectrum once they cross the Hubble horizon. Independent of the initial vacuum choice, a logarithmic and an oscillatory correction are present. Furthermore, the spectrum is proportional to $\eta^{-3 / 2}$ as it was for UV perturbations in (30), so this is a significant effect. Note, the factor $\sim\left(k \eta_{i}\right)^{-1 / 2}$ in (36) depends on the initial conditions given on super Hubble scales - this is not surprising since ambiguities are well known to arise on super Hubble scales 41]. The amplitude of the oscillations is not suppressed by small numbers. This is in contrast to the case of the minimal trans-Planckian approach to inflation where the amplitudes are of order $H / M_{p}$ 43.

Deep inside the Hubble horizon the spectrum of fluctuations is scale invariant according to (30), but due to IR fluctuations entering during the holographic phase, the spectrum becomes $k$-dependent according to (36) or (38) (even inside the Hubble horizon). For the oscillatory effects to be invisible in the CMBR, a period of inflation after the $p=\rho$ phase has to last for a sufficient number of e-foldings.

Were we would like to get an impression of the portion of the Hubble radius that must inflate at the end of the
Holographic phase in order to make the oscillations of the IR modes unobservable in the CMBR today. Consider an initial patch of the Universe during the holographic phase with Hubble radius $H^{-1}$. Now let the scale factor grow by a few orders of magnitude (say $10^{3}$ ). During this time the Hubble radius will grow as $H^{-1} \sim a^{3}$. An initial quantum fluctuation with physical wavelength of order the Hubble radius has $\lambda_{p h}(\eta) / r_{H}(\eta) \sim 1 / a(\eta)^{2}$, (since $\lambda_{p h} \sim a$ ). So, if inflation sets in after the scale factor has grown by the three orders of magnitude a patch with a radius of about $10^{-6} H^{-1}$ must inflate, and not simply a patch of size $H^{-1}$. Thus, it seems the inflationary phase in the Holographic Universe can not be much shorter than typical inflationary phases in standard cosmological models.

\section{VECTOR PERTURBATIONS IN THE $p=\rho$ ERA}

Vector perturbations (VP) have recently been studied in the context of bouncing cosmologies, due to their growing nature in a contracting Universe [44, 45]. However, if the fluid is stiff enough, VP will also grow in an expanding Universe (as was first pointed out in [46]). We will now examine them in the context of a $\rho=p$ Universe.

The most general perturbed metric including only VP is given by 40.

$$
\left(\delta g_{\mu \nu}\right)=-a^{2}\left(\begin{array}{cc}
0 & -S^{i} \\
-S^{i} & F_{, j}^{i}+F_{, i}^{j}
\end{array}\right)
$$


where the vectors $S$ and $F$ are divergenceless, that is $S^{i}{ }_{, i}=0$ and $F^{i}{ }_{i}=0$.

A gauge invariant VP can be defined as [44]

$$
\sigma^{i}=S^{i}+F^{i \prime}
$$

The most general perturbation of the energy momentum tensor including only VP is given by [4]

$$
\left(\delta T_{\beta}^{\alpha}\right)=\left(\begin{array}{cc}
0 & -(\rho+p) V^{i} \\
(\rho+p)\left(V^{i}+S^{i}\right) & p\left(\pi^{i}, j+\pi_{, i}{ }_{, i}\right)
\end{array}\right),
$$

where $\pi^{i}$ and $V^{i}$ are divergenceless. Furthermore $V^{i}$ is related to the perturbation in the 4 -velocity via

$$
\left(\delta u^{\mu}\right)=\left(\begin{array}{c}
0 \\
\frac{V^{i}}{a}
\end{array}\right)
$$

Gauge invariant quantities are given by

$$
\theta^{i}=V^{i}-F^{i \prime}
$$

and $\pi^{i}$.

From now on we work in Newtonian gauge where $F^{i}=0$ so that $\sigma$ coincides with $S$ and $\theta$ with $V$. Note that there is no residual gauge freedom after going to Newtonian gauge.

If we assume for simplicity $\pi^{i}=0$ (no anisotropic stress), the equations of motion for each Fourier mode $V_{k}$ and $S_{k}$ are solved by [4]

$$
S_{k}^{i}=\frac{C_{k}^{i}}{a^{2}}
$$

where $C_{k}^{i}$ is a constant and

$$
\begin{aligned}
V_{k}^{i} & =\frac{k_{4}^{2} k^{2}}{2(\rho+p) a^{2}} S_{k}^{i} \\
& \sim \frac{k^{2} C_{k}^{i}}{a^{1-3 w}} .
\end{aligned}
$$

Thus, in the stiff fluid dominated phase the metric perturbation is decreasing $S_{k}^{i}=\sim \eta^{-1}$ but $V_{k}^{i} \sim \eta$ is increasing. Nevertheless, the contribution to the perturbed energy momentum tensor (41) is still finite and, in fact, decreasing since

$$
\begin{aligned}
(p+\rho) V_{k}^{i} & \sim a^{-3(1+w)} a^{-1+3 w} \\
& \sim \eta^{-2}
\end{aligned}
$$

\section{A. Consequences}

The interpretation of this peculiar behavior of $\mathrm{VP}$ is as follows: in the holographic phase there is so much inertia in the matter sector that eddies expanding with the Universe are actually speeding up [4]. Since the energy density in the black hole fluid scales as $\rho \sim a^{-6}$ the contribution to the energy momentum tensor due to vorticities becomes more and more significant as the Universe expands (from Eq. (47), $\left.(p+\rho) V_{k}^{i} \sim a^{-4}\right)$. This results in a turbulent Universe at the end of the holographic phase. Note that the old arguments that ruled out turbulent cosmologies based on Nucleosynthesis, e.g. [48], do not apply here, since the Holographic Universe requires an inflationary phase.

Furthermore, a significant rotation always creates a significant shear [58]. It seems likely that this will produce a highly anisotropic Universe after the holographic phase. We speculate, that it is this back-reaction of VP that could put an end to the stiff fluid dominated epoch, since after some time the simple $p=\rho$ fluid description is no longer accurate. If this is the case, any subsequent inflationary phase has to resolve an isotropy problem, in addition to stretching UV perturbations to super Hubble scales.

\section{TENSOR PERTURBATIONS IN THE $p=\rho$ ERA}

Here we study gravitational waves in the Holographic Universe. Consider tensor metric fluctuations 31, 49, 50. about a classical cosmological background

$$
d s^{2}=a^{2}(\eta)\left[d \eta^{2}-\left(\delta_{i j}+h_{i j}\right) d x^{i} d x^{j}\right] .
$$

The gravitational waves are represented by the second rank tensor $h_{i j}(\eta, \mathbf{x})$. Expanding the Einstein-Hilbert action to second order in the fluctuating fields yields

$$
\delta_{2} S=\int d^{4} x \frac{a^{2}(\eta)}{2}\left[\left(h^{i}{ }_{j}\right)^{\prime}\left(h^{j}{ }_{i}\right)^{\prime}-\partial_{k}\left(h^{i}{ }_{j}\right) \partial^{k}\left(h^{j}{ }_{i}\right)\right] .
$$

Decomposing $h_{i j}$ into its two polarization states $e_{i j}^{+}$and $e_{i j}^{x}$ gives

$$
h_{i j}(\eta, \mathbf{x})=\mathrm{h}_{+}(\eta, \mathbf{x}) \mathrm{e}_{\mathrm{ij}}^{+}+\mathrm{h}_{\mathrm{x}}(\eta, \mathbf{x}) \mathrm{e}_{\mathrm{ij}}^{\mathrm{x}},
$$

where $h_{+}$and $h_{x}$ are coefficient functions. Decomposing $h^{s}, s \in\{+, x\}$, into its Fourier modes and defining $\mu_{k}^{s}=$ $a h_{k}^{s}$ such that the action for both $\mu_{k}^{s}$ takes canonical form, we arrive at the equations of motions

$$
\mu_{k}^{\prime \prime}+\left(k^{2}-\frac{a^{\prime \prime}}{a}\right) \mu_{k}=0
$$

where we suppressed the superscript $s$. Since, in the case of Holographic cosmology, $\sqrt{3} a=z$, the above equation has the same solution as the equation for the scalar perturbations (20). Thus the spectrum of gravitational waves behaves in the same way as the spectrum for scalar perturbations in sections IVA and IVB [59].

\section{CONCLUSION}

In this article we computed the spectrum of scalar and tensor metric perturbations in a Universe that is 
dominated by a stiff fluid with equation of state $p=\rho$ (e.g., the Holographic Universe). UV perturbations that stayed inside the Hubble horizon during the entire holographic phase exhibit a scale invariant spectrum. However, IR perturbations that enter the Horizon during this phase yield oscillatory and logarithmic corrections. Despite ambiguities related to quantum mechanical initial conditions, the results seem to be robust with respect to different choices of the initial vacuum. Because no such oscillations are observed on scales that enter the horizon today, we conclude that the required era of inflation after the holographic phase cannot be much shorter than it has to be in standard inflationary models of the Universe. That way, only the scale invariant UV part of the spectrum becomes observable.

Vector perturbations exhibit growing solutions during the holographic phase. These result in an anisotropic and turbulent Universe, if the holographic phase lasts long enough. We speculate that it is this back-reaction of vector perturbations that will ultimately put an end to the holographic phase.

It should be noted that the holographic Universe model is still in its infancy. The implementation of the much needed inflationary phase has yet to be achieved. Also, a better understanding of the microphysical properties of the black hole fluid and how it originates from an epoch of quantum gravity is desirable. Although the holographic cosmology proposal does not eliminate the need for a period of cosmological inflation, there is hope that it will help resolve the initial singularity.

\section{Acknowledgments}

We would like to thank R. Brandenberger for many useful discussions and comments on a draft of this work. It is a pleasure to thank T. Banks and W. Fischler for an important personal communication and J. Barrow for helpful discussions. We also thank Northeasthern University for its hospitality during PASCOS 04 and NathFest where this work was completed. DE is supported in part by the National Science Foundation under grant PHY-0094122 and by funds from Syracuse University.
[1] S. Kachru, R. Kallosh, A. Linde, J. Maldacena, L. McAllister and S. P. Trivedi, JCAP 0310, 013 (2003), arXiv:hep-th/0308055.

[2] J. Khoury, B. A. Ovrut, P. J. Steinhardt and N. Turok, Phys. Rev. D 64, 123522 (2001), arXiv:hep-th/0103239.

[3] P. J. Steinhardt and N. Turok, arXiv:hep-th/0111030

[4] R. H. Brandenberger and C. Vafa, Nucl. Phys. B 316, 391 (1989).

[5] S. Alexander, R. H. Brandenberger and D. Easson, Phys. Rev. D 62, 103509 (2000), arXiv:hep-th/0005212.

[6] R. Brandenberger, D. A. Easson and D. Kimberly, Nucl. Phys. B 623, 421 (2002) arXiv:hep-th/0109165.

[7] L. Randall and R. Sundrum, Phys. Rev. Lett. 83, 3370 (1999) arXiv:hep-ph/9905221.

[8] L. Randall and R. Sundrum, Phys. Rev. Lett. 83, 4690 (1999) arXiv:hep-th/9906064.

[9] G. Veneziano, Phys. Lett. B 265, 287 (1991).

[10] M. Gasperini and G. Veneziano, Astropart. Phys. 1, 317 (1993) arXiv:hep-th/9211021.

[11] J. E. Lidsey, D. Wands and E. J. Copeland, Phys. Rept. 337, 343 (2000) arXiv:hep-th/9909061.

[12] D. A. Easson, Int. J. Mod. Phys. A 16, 4803 (2001). arXiv:hep-th/0003086.

[13] S. M. Carroll, arXiv:hep-th/0011110

[14] D. A. Easson, arXiv:hep-th/0111055

[15] F. Quevedo, Class. Quant. Grav. 19, 5721 (2002) arXiv:hep-th/0210292.

[16] M. Gasperini and G. Veneziano, Phys. Rept. 373, 1 (2003) arXiv:hep-th/0207130.

[17] P. Brax and C. van de Bruck, Class. Quant. Grav. 20, R201 (2003) arXiv:hep-th/0303095.

[18] T. Battefeld and S. Watson, JCAP 0406, 001 (2004) arXiv:hep-th/0403075.

[19] D. N. Spergel et al., Astrophys. J. Suppl. 148, 175 (2003) arXiv:astro-ph/0302209.
[20] G. 't Hooft, arXiv:gr-qc/9310026

[21] L. Susskind, J. Math. Phys. 36, 6377 (1995) arXiv:hep-th/9409089.

[22] T. Banks, W. Fischler and L. Mannelli, arXiv:hep-th/0408076

[23] T. Banks and W. Fischler, arXiv:hep-th/0405200

[24] T. Banks and W. Fischler, arXiv:hep-th/0310288

[25] T. Banks and W. Fischler, arXiv:hep-th/0212113

[26] T. Banks and W. Fischler, arXiv:hep-th/0111142

[27] L. Fernandez-Jambrina and L. M. Gonzalez-Romero, J. Math. Phys. 45, 2113 (2004) arXiv:gr-qc/0405013.

[28] M. K. Mak and T. Harko, Int. J. Mod. Phys. D 13, 273 (2004) arXiv:gr-qc/0311050.

[29] S. S. Yazadjiev, Mod. Phys. Lett. A 18, 471 (2003).

[30] S. S. Yazadjiev, Phys. Rev. D 66, 024031 (2002) arXiv:gr-qc/0203041.

[31] V. Sahni, M. Sami and T. Souradeep, Phys. Rev. D 65, 023518 (2002) arXiv:gr-qc/0105121.

[32] M. K. Mak and T. Harko, Europhys. Lett. 56, 762 (2001) arXiv:gr-qc/0107004.

[33] R. S. R. and B. S. K., Gen. Rel. Grav. 28, 27 (1996).

[34] J. P. Abreu, P. Crawford and J. P. Mimoso, Prepared for Spanish Relativity Meetings (ERE), Mao, Menorca, 12-14 Sep 1994

[35] L. K. Patel and N. Dadhich, arXiv:gr-qc/9302001

[36] J. Pullin, Astrophys. Space Sci. 164, 309 (1990).

[37] R. J. Gleiser, M. C. Diaz and R. D. Grosso, Class. Quant. Grav. 5, 989 (1988).

[38] L. P. Grishchuk, Ann. NY. Acad. Sci. 302, 439 (1977).

[39] G. Veneziano, JCAP 0403, 004 (2004) arXiv:hep-th/0312182.

[40] V. F. Mukhanov, H. A. Feldman and R. H. Brandenberger, Phys. Rept. 215, 203 (1992).

[41] M. Giovannini, Class. Quant. Grav. 20, 5455 (2003) arXiv:hep-th/0308066. 
[42] R. Brandenberger and P. M. Ho, Phys. Rev. D 66, 023517 (2002) [AAPPS Bull. 12N1, 10 (2002)] arXiv:hep-th/0203119.

[43] J. Martin and R. Brandenberger, Phys. Rev. D 68, 063513 (2003) arXiv:hep-th/0305161.

[44] T. J. Battefeld and R. Brandenberger, arXiv:hep-th/0406180

[45] M. Giovannini, arXiv:hep-th/0407124

[46] John D. Barrow Mon. Not. Roy. astr. Soc. , 179, 47P-49P (1977).

[47] J. M. Bardeen, Phys. Rev. D22, 1882 (1980).

[48] John D. Barrow Mon. Not. Roy. astr. Soc. , 178, 625-650 (1977).

[49] R. H. Brandenberger, Lect. Notes Phys. 646, 127 (2004) arXiv:hep-th/0306071.

[50] V. Sahni, Phys. Rev. D 42, 453 (1990).

[51] M. Giovannini, Phys. Rev. D 58, 083504 (1998) arXiv:hep-ph/9806329.

[52] M. Giovannini, Phys. Rev. D 60, 123511 (1999) arXiv:astro-ph/9903004.
[53] M. Giovannini, Class. Quant. Grav. 16, 2905 (1999) arXiv:hep-ph/9903263.

[54] For reviews see e.g. 11, 12, 13, 14, 15, 16, 17, 18].

[55] The idea of a stiff fluid present in the early Universe can probably be attributed to Zeldovich. For a simple analysis of the perturbation spectra see e.g., [38] and the references therein.

[56] See however arguments in favor of a scale invariant spectrum based on symmetry in [24].

[57] Our analysis assumes that the techniques of local field theory are applicable to describe the origin of fluctuations. This may not be justified for a theory based on holography. We thank T. Banks and W. Fischler for raising this point.

[58] We thank J. Barrow for useful comments on this point.

[59] The presence of logarithmic terms in the spectra of tensor modes agrees with the results found by M. Giovannini in 51]-53] 